\title{
Stability analysis of a certain class of difference equations by using KAM theory
}

\section{Senada Kalabušićc ${ }^{*}$ (D), Emin Bešo ${ }^{1}$, Naida Mujić ${ }^{2}$ and Esmir Pilav ${ }^{1}$}

\section{"Correspondence:}

kalabusicsenada06@gmail.com 'Department of Mathematics, Faculty of Science, University of Sarajevo, Sarajevo, Bosnia and Herzegovina

Full list of author information is available at the end of the article

\begin{abstract}
By using KAM theory we investigate the stability of equilibrium points of the class of difference equations of the form $x_{n+1}=\frac{f\left(x_{n}\right)}{x_{n-1}}, n=0,1, \ldots, f:(0,+\infty) \rightarrow(0,+\infty), f$ is sufficiently smooth and the initial conditions are $x_{-1}, x_{0} \in(0,+\infty)$. We establish when an elliptic fixed point of the associated map is non-resonant and non-degenerate, and we compute the first twist coefficient $\alpha_{1}$. Then we apply the results to several difference equations.
\end{abstract}

Keywords: Area-preserving map; Difference equation; KAM theory; Periodic orbit

\section{Introduction and preliminaries}

By using KAM (Kolmogorov-Arnold-Mozer) theory we investigate the stability properties of solutions of the following class of second-order difference equations:

$$
x_{n+1}=\frac{f\left(x_{n}\right)}{x_{n-1}}, \quad n=0,1, \ldots
$$

where $f$ is sufficiently smooth, $f:(0,+\infty) \rightarrow(0,+\infty)$, and the initial conditions are $x_{-1}, x_{0} \in(0,+\infty)$.

Equation (1) is considered in the book [18] where $f:(0,+\infty) \rightarrow(0,+\infty)$ and the initial conditions are $x_{-1}, x_{0} \in(0,+\infty)$. In particular, several open problems and conjectures concerning the possible choice of the function $f$, for which the difference equation (1) is globally periodic, are listed. In [25] the answers to some open problems and conjectures listed in the book [18] are given. Precisely, for the cases $p \leq 5$, necessary and sufficient conditions on $f$ for all solutions to be periodic with period $p$ are found.

The well-known difference equation of the form (1) is Lyness' equation

$$
x_{n+1}=\frac{x_{n}+\beta}{x_{n-1}}, \quad n=0,1, \ldots
$$

Several authors have studied the Lyness equation (2) and have obtained numerous results concerning the stability of equilibrium, non-existence of solutions that converge to the equilibrium point, the existence of invariants, etc.; see [2, 14, 15, 17, 19, 35]. See [16] for the application of the KAM theory to Lyness equation (2). See also [3, 4, 6] for the results on the feasible periods for solutions of (2) and the existence of non-periodic solutions

(c) The Author(s) 2019. This article is distributed under the terms of the Creative Commons Attribution 4.0 International License (http://creativecommons.org/licenses/by/4.0/), which permits unrestricted use, distribution, and reproduction in any medium, provided you give appropriate credit to the original author(s) and the source, provide a link to the Creative Commons license, and indicate if changes were made. 
of (2). See [20,21] for the results on the stability of Lyness equation (2) with period two and period three coefficients. These proofs were based on the construction of the corresponding Lyapunov functions associated with the invariants of the equation. See also [21] for the results on the stability of Lyness equation with period two coefficient by using KAM theory.

In $[1,7]$ authors consider the rational second-order difference equation

$$
x_{n+1}=\frac{\alpha}{\left(1+x_{n}\right) x_{n-1}}, \quad n=0,1,2, \ldots
$$

as a special case of the rational difference equation

$$
x_{n+1}=\frac{\alpha+\beta x_{n} x_{n-1}+\gamma x_{n-1}}{A+B x_{n} x_{n-1}+C x_{n-1}}, \quad n=0,1,2, \ldots,
$$

with nonnegative parameters and with arbitrary nonnegative initial conditions such that the denominator is always positive. Equation (3) is of the form (1). Equation (3) possesses the following invariant:

$$
x_{n-1}+x_{n}+x_{n-1} x_{n}+\alpha\left(\frac{1}{x_{n-1}}+\frac{1}{x_{n}}\right)=\text { constant }, \quad \forall n \geq 0 .
$$

See [1]. Equation (3) has a unique positive equilibrium point, and the characteristic equation of the linearized equation of (3) about the equilibrium point has two complex conjugate roots on $|\lambda|=1$. Several conjectures and open problems concerning the stability of the equilibrium point as well as the periodicity of solutions are listed, see [1]. For a more general case of Equation (3), see [10].

The following equation, which is of the form (1):

$$
y_{n+1}=\frac{\alpha y_{n}^{2}}{\left(1+y_{n}\right) y_{n-1}}, \quad n=1,2, \ldots
$$

where $\alpha$ is a parameter, is known as May's host parasitoid equation, see [22]. In [22] the authors investigated the corresponding map known as May's map. More precisely, they investigated the following system of rational difference equations:

$$
\begin{aligned}
& u_{n+1}=\frac{\alpha u_{n}}{1+\beta v_{n}}, \\
& v_{n+1}=\frac{\beta u_{n} v_{n}}{1+\beta v_{n}}, \quad n=0,1,2, \ldots,
\end{aligned}
$$

where $\alpha$ and $\beta$ are positive numbers and initial conditions $u_{0}$ and $v_{0}$ are arbitrary positive numbers. When $\alpha \in(1,+\infty)$ and $\beta \in(0, \infty)$ this system is a special case of May's host parasitoid model. The change of variables $x_{n}=\beta u_{n}$ and $y_{n}=\beta v_{n}$ reduces System (5) to

$$
\begin{aligned}
x_{n+1} & =\frac{\alpha x_{n}}{1+y_{n}}, \\
y_{n+1} & =\frac{x_{n} y_{n}}{1+y_{n}}, \quad n=0,1,2, \ldots .
\end{aligned}
$$


By eliminating $x_{n}$ from the right-hand side, System (6) reduces to Equation (4). In [23, 24, 33] it was asserted that the positive equilibrium $\left(\frac{\alpha}{\beta}, \frac{\alpha-1}{\beta}\right)$ of System (5) is not asymptotically stable. In [22] it was proved that this is the case, and then, by employing KAM theory, the authors showed that the positive equilibrium of System (5) is stable. See [30] for results on periodic solutions.

In [12] authors analyzed a certain class of difference equations governed by two parameters

$$
x_{n+1}=\frac{x_{n}^{k}+a}{x_{n}^{p} x_{n-1}},
$$

where $k, p$, and $a$ are positive and the initial conditions $x_{0}, x_{1}$ are positive. They fixed the value of $a$ as $a=\left(2^{k-p-2}-1\right) / 2^{k}$ and gave an essentially complete description of the global behavior of solutions in the first quadrant. They showed how Equation (7) leads to diffeomorphism $F$ and showed that, for certain parameter value, all such $F$ share four key properties. One of these is that $F$ has precisely two fixed points. Then they showed that an "upper" fixed point is hyperbolic, and they showed by using KAM theory that, by further restricting $k$ and $l$, the origin becomes a neutrally stable elliptic point. Also, they showed that outside a compact neighborhood of the origin containing the two fixed points, all points tend to infinity at an exponential rate under the iterates of $F$ and $F^{-1}$ and two branches of the eigenmanifolds of the hyperbolic point intersect at a homoclinic point. Notice that Equation (7) has the form (1).

In [28] authors considered the following difference equation:

$$
x_{n+1}=\frac{A x_{n}^{3}+B}{a x_{n-1}}, \quad n=0,1, \ldots
$$

where the parameters $A, B, a$ and the initial conditions $x_{-1}, x_{0}$ are positive numbers. They employed KAM theory to investigate stability property of the positive elliptic equilibrium. Equation (8) is a special case of the following equation:

$$
x_{n+1}=\frac{A x_{n}^{k}+B}{a x_{n-1}}, \quad n=0,1, \ldots
$$

See [19]. See [13] for the equation

$$
x_{n+1}=\frac{A x_{n}^{2}+F}{e x_{n-1}}, \quad n=0,1, \ldots
$$

In [8] authors considered the following difference equation:

$$
x_{n+1}=\frac{A+B x_{n}+x_{n}^{2}}{\left(1+D x_{n}\right) x_{n-1}}, \quad n=0,1, \ldots
$$

They employed KAM theory to investigate stability property of the positive elliptic equilibrium.

Notice that all of these equations are of the form (1).

By using the methods of algebraic and projective geometry in $[4,5]$, the authors studied algebraic generalization of Lyness difference equation. More precisely, they analyzed 
global behavior of the following difference equations:

$$
\begin{aligned}
& u_{n+2} u_{n}=a+b u_{n+1}+u_{n+1}^{2}, \quad u_{n+2} u_{n}=\frac{a+b u_{n+1}+c u_{n+1}^{2}}{c+u_{n+1}} \text { and } \\
& u_{n+2} u_{n}=\frac{a+b u_{n+1}+c u_{n+1}^{2}}{c+d u_{n+1}+u_{n+1}^{2}}
\end{aligned}
$$

They obtained very precise description of complicated global behavior which includes finding the possible periods of all solutions, proving the existence of chaotic solutions through conjugation of maps, and so forth. These methods were first used by Zeeman in [35] for the study of Lyness equation. Notice that each of these equations has the form (1).

Motivated by all these results, we consider any real function $f$ of one real variable which is sufficiently smooth and $f:(0,+\infty) \rightarrow(0,+\infty)$, and then we consider Equation (1). In Sect. 2 we show how (1) leads to diffeomorphisms $T$ and $F$. We prove some properties of the map $T$, and we establish the condition under which a fixed point $(\bar{x}, \bar{x})$ of the map $T$, in $(u, v)$ coordinates $(0,0)$, is an elliptic fixed point, where $\bar{x}$ is an equilibrium point of Equation (1). In Sect. 3 we compute the first twist coefficient $\alpha_{1}$, and we establish when an elliptic fixed point of the map $T$ is non-resonant and non-degenerate. In Sect. 4 we apply our results to several difference equations of the form (1), and we visualize the behavior of solutions for some values of the corresponding parameters.

\section{Logarithmic coordinate change and area-preserving property}

We may write Equation (1) as a map $T:(0,+\infty)^{2} \rightarrow(0,+\infty)^{2}$ by setting

$$
u_{n}=x_{n-1}, \quad v_{n}=x_{n}, \quad T\left(\begin{array}{l}
u \\
v
\end{array}\right)=\left(\begin{array}{c}
v \\
\frac{f(v)}{u}
\end{array}\right) .
$$

The fixed point $(\bar{u}, \bar{v})$ of the map $T$ satisfies the following:

$$
\bar{u}=\bar{v} \quad \text { and } \quad \frac{f(\bar{v})}{\bar{u}}=\bar{v}
$$

which implies

$$
\bar{u}^{2}=f(\bar{u}) .
$$

Note that $\bar{u}=\bar{v}=\bar{x}$, where $\bar{x}$ is the equilibrium point of Equation (1).

We will assume that all maps are sufficiently smooth to justify subsequent calculations. The map $T$ itself must be diffeomorphism of $(0,+\infty)^{2}$, and therefore we assume that this is the case. The inverse of $T$ is given by

$$
T^{-1}\left(\begin{array}{l}
u \\
v
\end{array}\right)=\left(\begin{array}{c}
u \\
\frac{f(u)}{v}
\end{array}\right)
$$

The planar map $F$ is area-preserving or conservative if the map $F$ preserves area of the planar region under the forward iterate of the map, see [11, 19, 32]. A differentiable map $F$ is area-preserving if and only if the absolute value of determinant of the Jacobian matrix 
of the map $F$ is equal to 1 , that is, $\left|\operatorname{det} J_{F}(x, y)\right|=1$ at every point $(x, y)$ of the domain of $F$, see $[11,32]$.

We claim that map (9) is exponentially equivalent to an area-preserving map, see [16]. Let

$$
E(u, v)=\left(\bar{x} e^{u}, \bar{x} e^{v}\right)^{T} .
$$

Then

$$
E^{-1}(x, y)=\left(\ln \frac{x}{\bar{x}}, \ln \frac{y}{\bar{x}}\right)^{T}
$$

and if we set $F(u, v)=E^{-1} \circ T \circ E(u, v)$, where $\circ$ denotes composition of functions, then we obtain a new mapping $F$, which is given by

$$
F(u, v)=E^{-1} \circ T \circ E(u, v)=\left(\begin{array}{c}
v \\
\ln \left(f\left(e^{v} \bar{x}\right)\right)-2 \ln (\bar{x})-u
\end{array}\right) .
$$

The map $F$ is defined on all of $\mathbf{R}^{2}$. In fact, since $T$ was a diffeomorphism of the open first quadrant $Q$ and since $E$ is a diffeomorphism of $\mathbf{R}^{2}$ onto $Q, F$ is a diffeomorphism of $\mathbf{R}^{2}$ onto itself.

In the study of area-preserving maps, symmetries play an important role since they yield special dynamic behavior. A transformation $R$ of the plane is said to be a time reversal symmetry for $T$ if $R^{-1} \circ T \circ R=T^{-1}$, meaning that applying the transformation $R$ to the map $T$ is equivalent to iterating the map backwards in time. If the time reversal symmetry $R$ is an involution, i.e., $R^{2}=i d$, then the time reversal symmetry condition is equivalent to $R \circ T \circ R=T^{-1}$, and $\mathrm{T}$ can be written as the composition of two involutions $T=I_{1} \circ I_{0}$, with $I_{0}=R$ and $I_{1}=T \circ R$. Note that if $I_{0}=R$ is a reversor, then so is $I_{1}=T \circ R$. Also, the $j$ th involution, defined as $I_{j}:=T^{j} \circ R$, is also a reversor.

Similar to the proof of Theorem 2.1 in [12], we prove some properties of the map $F$ in the following lemma.

Lemma 1 Assume $f \in C^{1}[(0,+\infty),(0,+\infty)], f(\bar{x})=\bar{x}^{2}$, and $\bar{x}>0$, then Fshares the following properties:

(a) F has the origin as a fixed point;

(b) $F$ is globally area-preserving;

(c) F satisfies a time-reversing, mirror image, symmetry condition;

(d) All fixed points of $F$ are located on the diagonal in the first quadrant.

Proof Assertion (a) is immediate. The Jacobian matrix of the map $F$ is

$$
J_{F}(u, v)=\left(\begin{array}{cc}
0 & 1 \\
-1 & \frac{e^{v} \bar{x} f^{\prime}\left(e^{v} \bar{x}\right)}{f\left(e^{v} \bar{x}\right)}
\end{array}\right),
$$

and so $\operatorname{det} J_{F}(u, v)=1$. To explain (c), let $R(x, y)=(y, x)$ which is reflection about the diagonal. An easy calculation shows that $R^{2}=i d$, and the map $F$ will satisfy $F \circ R \circ F=R$. This 
equation may be rewritten as $R \circ F=F^{-1} \circ R$. For the final assertion (d), it is easier to work with the original form of our function $T$.

A fixed point $(\bar{x}, \bar{x})$ is an elliptic point of an area-preserving map if the eigenvalues of $J_{T}(\bar{x}, \bar{y})$ form a purely imaginary, complex conjugate pair $\lambda, \bar{\lambda}$, see $[11,19]$. The following lemma holds.

Lemma 2 Assume that $f(\bar{x})=\bar{x}^{2}$ and $\bar{x}>0$. Then the map $T$ in $(x, y)$ coordinates has an elliptic fixed point $(\bar{x}, \bar{x})$ if and only if $\left|f^{\prime}(\bar{x})\right|<2 \bar{x}$. In the $(u, v)$ coordinates, the corresponding fixed point is $(0,0)$.

Proof For the fixed points in $(x, y)$ coordinates, solving $y=x$ and $f(x)=x^{2}$ yields the fixed point $(\bar{x}, \bar{x})$ where $f(\bar{x})=\bar{x}^{2}$. Evaluating the Jacobian matrix of $T$ at $(\bar{x}, \bar{x})$ by using $f(\bar{x})=\bar{x}^{2}$ gives

$$
J_{T}(\bar{x}, \bar{x})=\left(\begin{array}{cc}
0 & 1 \\
-\frac{f(\bar{x})}{\bar{x}^{2}} & \frac{f^{\prime}(\bar{x})}{\bar{x}}
\end{array}\right)=\left(\begin{array}{cc}
0 & 1 \\
-1 & \frac{f^{\prime}(\bar{x})}{\bar{x}}
\end{array}\right) .
$$

We obtain that the eigenvalues of $J_{T}(\bar{x}, \bar{x})$ are $\lambda, \bar{\lambda}$ where

$$
\lambda=\frac{f^{\prime}(\bar{x})-i \sqrt{4 \bar{x}^{2}-\left[f^{\prime}(\bar{x})\right]^{2}}}{2 \bar{x}} .
$$

Since $|\lambda|=1$, we have that $(\bar{x}, \bar{x})$ is an elliptic fixed point if and only if $\left|f^{\prime}(\bar{x})\right|<2 \bar{x}$. Under the logarithmic coordinate change $(x, y) \rightarrow(u, v)$ the fixed point $(\bar{x}, \bar{x})$ becomes $(0,0)$.

\section{The KAM theory and Birkhoff normal form}

The stability of an elliptic fixed point of nonlinear area-preserving map cannot be determined solely from linearization, and the effects of the nonlinear terms in local dynamics must be accounted for. This task is facilitated by simplifying the nonlinear terms through appropriate coordinate transformations into Birkhoff normal form.

Consider a smooth, area-preserving map $(u, v) \rightarrow F(u, v)$ of the plane that has $(0,0)$ as an elliptic fixed point, and let $\lambda$ be an eigenvalue of $J_{F}(0,0)$. By putting the linear part of such a map into Jordan canonical form, by making an appropriate change of variables, we can represent the map in the form

$$
\left(\begin{array}{c}
\tilde{u} \\
\tilde{v}
\end{array}\right) \rightarrow\left(\begin{array}{cc}
\operatorname{Re}(\lambda) & -\operatorname{Im}(\lambda) \\
\operatorname{Im}(\lambda) & \operatorname{Re}(\lambda)
\end{array}\right)\left(\begin{array}{c}
\tilde{u} \\
\tilde{v}
\end{array}\right)+\tilde{F}\left(\begin{array}{c}
\tilde{u} \\
\tilde{v}
\end{array}\right) .
$$

By using complex coordinates $z, \bar{z}=\tilde{u} \pm i \tilde{v}$ map (11) leads to the complex form

$$
z \rightarrow \lambda z+\xi_{20} z^{2}+\xi_{11} z \bar{z}+\xi_{02} \bar{z}^{2}+\xi_{30} z^{3}+\xi_{21} z^{2} \bar{z}+\xi_{12} z \bar{z}^{2}+\xi_{03} \bar{z}^{3}+O\left(|z|^{4}\right) .
$$

Assume that the eigenvalue $\lambda$ of the elliptic fixed point satisfies the non-resonance condition $\lambda^{k} \neq 1$ for $k=1, \ldots, q$, for some $q \geq 4$. By Lemma 15.37 [11] there exist new canonical complex coordinates $(\zeta, \bar{\zeta}$ ) relative to which mapping (12) takes the normal form (Birkhoff normal form)

$$
\zeta \rightarrow \lambda \zeta e^{i \alpha(\zeta \bar{\zeta})}+g(\zeta, \bar{\zeta})
$$


in a neighborhood of the elliptic fixed point, where $\alpha(\zeta \bar{\zeta})=\alpha_{1}|\zeta|^{2}+\cdots+\alpha_{s}|\zeta|^{2 s}$ is a real polynomial, $s=\left[\frac{q}{2}\right]-1$, and $g$ vanishes with its derivatives up to order $q-1$ at $\zeta=\bar{\zeta}=0$. The square brackets denote the largest integer in $q / 2$. The numbers $\alpha_{1}, \ldots, \alpha_{s}$ are called twist coefficients.

Consider an invariant annulus $a<|\zeta|<b$ in a neighborhood of an elliptic fixed point $(0,0)$. It is easy to see that the normal form approximation $\zeta \rightarrow \lambda \zeta e^{i \alpha(\zeta \bar{\zeta})}$ leaves invariant all circles $|\zeta|=$ const. This map is called a twist mapping. It is easy to describe the dynamics of the twist map: the orbits are simple rotations on these circles. Also note that if at least one of the twist coefficients $\alpha_{j}$ is nonzero, then the angle of rotation is not constant. Applying KAM-theory (Moser's twist map theorem [9, 27, 29, 31]) it follows that if a system is close enough to a twist mapping with rotation angle varying with the radius, then still infinitely many of the invariant circles survive the perturbation. By [29], p. 245, the rotation angles of these circles are only badly approximable by rational numbers. According to KAMtheory there exist states close enough to the fixed point, which are enclosed by an invariant curve. Within these gaps, one finds, in general, orbits of hyperbolic and elliptic periodic points. These facts cannot be deduced from computer pictures. By continuity arguments the interior of such a closed invariant curve will then map onto itself. The same is true for a state within an annulus enclosed between two such curves.

The KAM theorem requires that the elliptic fixed point be non-resonant and nondegenerate. Note that, for $q=4$, the non-resonance condition $\lambda^{k} \neq 1$ requires that $\lambda \neq \pm 1$ or $\pm i$. The above normal form yields the approximation

$$
\zeta \rightarrow \lambda \zeta+c_{1} \zeta^{2} \bar{\zeta}+O\left(|\zeta|^{4}\right)
$$

with $c_{1}=i \lambda \alpha_{1}$ and $\alpha_{1}$ being the first twist coefficient. We will call an elliptic fixed point non-degenerate if $\alpha_{1} \neq 0$.

The following is a consequence of Lemma 15.37 [11] and Moser's twist map theorem [9, $11,27,29]$.

Theorem 1 Let $F: \mathbb{R}^{2} \rightarrow \mathbb{R}^{2}$ be an area-preserving diffeomorphism and $(\bar{x}, \bar{y})$ be an elliptic fixed point. Assume that $\alpha_{1} \neq 0$. Then there exist periodic points of $F$ with arbitrarily large period in every neighborhood of $(\bar{x}, \bar{y})$. In addition, if $\lambda \neq \pm 1$ or $\pm i$, then the $(\bar{x}, \bar{y})$ is a stable fixed point.

In the sequel we set

$$
f_{1}:=f^{\prime}(\bar{x}), \quad f_{2}:=f^{\prime \prime}(\bar{x}) \quad \text { and } \quad f_{3}:=f^{\prime \prime \prime}(\bar{x})
$$

We prove the following theorem.

Theorem 2 Assume that $\left|f^{\prime}(\bar{x})\right|<2 \bar{x}$, where $\bar{x}$ is an equilibrium point of Equation (1). The elliptic fixed point $(0,0)$ of the map $F$, in the $(u, v)$ coordinates, is always non-degenerate. It is non-resonant if and only if

$$
f_{3} \neq \frac{f_{2}\left(f_{2}+6\right) \bar{x}^{4}+f_{1}\left(f_{2}\left(2 f_{2}-1\right)+2\right) \bar{x}^{3}-4 f_{1}^{2}\left(f_{2}+1\right) \bar{x}^{2}-f_{1}^{3} f_{2} \bar{x}+2 f_{1}^{4}}{\bar{x}^{3}\left(f_{1}-2 \bar{x}\right)\left(\bar{x}+f_{1}\right)} .
$$


Proof To compute the first twist coefficient $\alpha_{1}$, we follow the procedure in [9]. Let $F$ be the function defined by

$$
F\left(\begin{array}{l}
u \\
v
\end{array}\right)=\left(\begin{array}{c}
v \\
\log \left(f\left(e^{v} \bar{x}\right)\right)-2 \log (\bar{x})-u
\end{array}\right) .
$$

The Jacobian matrix of $F$ at $(u, v)$ is given by $(10)$. At $(0,0), J_{F}(u, v)$ has the form

$$
J_{0}=J_{F}(0,0)=\left(\begin{array}{cc}
0 & 1 \\
-1 & \frac{f_{1}}{\bar{x}}
\end{array}\right) .
$$

The eigenvalues of (14) are $\lambda$ and $\bar{\lambda}$ where

$$
\lambda=\frac{f_{1}-i \sqrt{4 \bar{x}^{2}-f_{1}^{2}}}{2 \bar{x}} .
$$

It is easy to see that

$$
\begin{aligned}
& \lambda^{2}=\frac{f_{1}^{2}}{2 \bar{x}^{2}}-\frac{i f_{1} \sqrt{4 \bar{x}^{2}-f_{1}^{2}}}{2 \bar{x}^{2}}-1, \\
& \lambda^{3}=\frac{f_{1}^{3}}{2 \bar{x}^{3}}-\frac{i f_{1}^{2} \sqrt{4 \bar{x}^{2}-f_{1}^{2}}}{2 \bar{x}^{3}}-\frac{3 f_{1}}{2 \bar{x}}+\frac{i \sqrt{4 \bar{x}^{2}-f_{1}^{2}}}{2 \bar{x}}, \\
& \lambda^{4}=\frac{f_{1}^{4}}{2 \bar{x}^{4}}-\frac{i f_{1}^{3} \sqrt{4 \bar{x}^{2}-f_{1}^{2}}}{2 \bar{x}^{4}}-\frac{2 f_{1}^{2}}{\bar{x}^{2}}+\frac{i f_{1} \sqrt{4 \bar{x}^{2}-f_{1}^{2}}}{\bar{x}^{2}}+1,
\end{aligned}
$$

from which it follows that $\lambda^{k} \neq 1$ for $k=1,2,3,4$.

Then we have that

$$
F\left(\begin{array}{l}
u \\
v
\end{array}\right)=J_{F}(0,0)\left(\begin{array}{l}
u \\
v
\end{array}\right)+F_{1}\left(\begin{array}{l}
u \\
v
\end{array}\right)
$$

where

$$
F_{1}\left(\begin{array}{l}
u \\
v
\end{array}\right)=\left(\begin{array}{c}
0 \\
-\frac{f_{1} v}{\bar{x}}+\log \left(f\left(e^{v} \bar{x}\right)\right)-2 \log (\bar{x})
\end{array}\right) .
$$

By using eigenvectors

$$
\mathbf{p}=\left(\frac{f_{1}-i \sqrt{4 \bar{x}^{2}-f_{1}^{2}}}{2 \bar{x}}, 1\right)
$$

and the associated matrix

$$
P=\frac{1}{\sqrt{D}}\left(\begin{array}{cc}
\frac{f_{1}}{2 \bar{x}} & -\frac{\sqrt{4 \bar{x}^{2}-f_{1}^{2}}}{2 \bar{x}} \\
1 & 0
\end{array}\right), \quad D=\frac{\sqrt{4 \bar{x}^{2}-f_{1}^{2}}}{2 \bar{x}}
$$

with determinant 1 , we change coordinates

$$
\left(\begin{array}{l}
\tilde{u} \\
\tilde{u}
\end{array}\right)=P^{-1}\left(\begin{array}{l}
u \\
v
\end{array}\right)=\sqrt{D}\left(\begin{array}{cc}
0 & 1 \\
-\frac{2 \bar{x}}{\sqrt{4 \bar{x}^{2}-f_{1}^{2}}} & \frac{f_{1}}{\sqrt{4 \bar{x}^{2}-f_{1}^{2}}}
\end{array}\right)\left(\begin{array}{l}
u \\
v
\end{array}\right)
$$

and bring the linear part into Jordan normal form. 
The system in the new coordinates becomes

$$
\left(\begin{array}{c}
\tilde{u} \\
\tilde{v}
\end{array}\right) \rightarrow\left(\begin{array}{cc}
\operatorname{Re}(\lambda) & -\operatorname{Im}(\lambda) \\
\operatorname{Im}(\lambda) & \operatorname{Re}(\lambda)
\end{array}\right)\left(\begin{array}{c}
\tilde{u} \\
\tilde{v}
\end{array}\right)+F_{2}\left(\begin{array}{c}
\tilde{u} \\
\tilde{v}
\end{array}\right),
$$

where

$$
\begin{aligned}
F_{2}\left(\begin{array}{l}
\tilde{u} \\
\tilde{v}
\end{array}\right) & =\left(\begin{array}{l}
g_{1}(\tilde{u}, \tilde{v}) \\
g_{2}(\tilde{u}, \tilde{v})
\end{array}\right)=P^{-1} F_{1}\left(P\left(\begin{array}{c}
\tilde{u} \\
\tilde{v}
\end{array}\right)\right) \\
& =\left(\begin{array}{c}
\sqrt{D}\left(\log \left(f\left(\bar{x} e^{\frac{\tilde{u}}{\sqrt{D}}}\right)\right)-2 \log (\bar{x})\right)-\frac{f_{1} \tilde{u}}{\bar{x}} \\
\frac{f_{1}\left(\sqrt{D} \bar{x}\left(\log \left(f\left(\bar{x} \frac{\tilde{u}}{\sqrt{D}}\right)\right)-2 \log (\bar{x})\right)-f_{1} \tilde{u}\right)}{\bar{x} \sqrt{4 \bar{x}^{2}-f_{1}^{2}}}
\end{array}\right) .
\end{aligned}
$$

One can now pass to the complex coordinates $z, \bar{z}=\tilde{u} \pm i \tilde{v}$ to obtain the complex form of the system

$$
z \rightarrow \lambda z+\xi_{20} z^{2}+\xi_{11} z \bar{z}+\xi_{02} \bar{z}^{2}+\xi_{30} z^{3}+\xi_{21} z^{2} \bar{z}+\xi_{12} z \bar{z}^{2}+\xi_{03} \bar{z}^{3}+O\left(|z|^{4}\right) .
$$

A tedious symbolic computation done with package Mathematica yields

$$
\begin{aligned}
\xi_{20} & =\frac{1}{8}\left\{\left(g_{1}\right)_{\tilde{u} \tilde{u}}-\left(g_{1}\right)_{\tilde{v} \tilde{v}}+2\left(g_{2}\right)_{\tilde{u} \tilde{v}}+i\left[\left(g_{2}\right)_{\tilde{u} \tilde{u}}-\left(g_{2}\right)_{\tilde{v} \tilde{v}}-2\left(g_{1}\right)_{\tilde{u} \tilde{v}}\right]\right\} \\
& =\frac{\left(\sqrt{4 \bar{x}^{2}-f_{1}^{2}}+i f_{1}\right)\left(f_{2} \bar{x}^{2}+f_{1} \bar{x}-f_{1}^{2}\right)}{4 \sqrt{2} \bar{x}^{3 / 2}\left(4 \bar{x}^{2}-f_{1}^{2}\right)^{3 / 4}}, \\
\xi_{11} & =\frac{1}{4}\left\{\left(g_{1}\right)_{\tilde{u} \tilde{u}}+\left(g_{1}\right)_{\tilde{v} \tilde{v}}+i\left[\left(g_{2}\right)_{\tilde{u} \tilde{u}}+\left(g_{2}\right)_{\tilde{v} \tilde{v}}\right]\right\}=\frac{\left(\sqrt{4 \bar{x}^{2}-f_{1}^{2}}+i f_{1}\right)\left(f_{2} \bar{x}^{2}+f_{1} \bar{x}-f_{1}^{2}\right)}{2 \sqrt{2} \bar{x}^{3 / 2}\left(4 \bar{x}^{2}-f_{1}^{2}\right)^{3 / 4}}, \\
\xi_{02} & =\frac{1}{8}\left\{\left(g_{1}\right)_{\tilde{u} \tilde{u}}-\left(g_{1}\right)_{\tilde{v} \tilde{v}}-2\left(g_{2}\right)_{\tilde{u} \tilde{v}}+i\left[\left(g_{2}\right)_{\tilde{u} \tilde{u}}-\left(g_{2}\right)_{\tilde{v} \tilde{v}}+2\left(g_{1}\right)_{\tilde{u} \tilde{v}}\right]\right\} \\
& =\frac{\left(\sqrt{4 \bar{x}^{2}-f_{1}^{2}}+i f_{1}\right)\left(f_{2} \bar{x}^{2}+f_{1} \bar{x}-f_{1}^{2}\right)}{4 \sqrt{2} \bar{x}^{3 / 2}\left(4 \bar{x}^{2}-f_{1}^{2}\right)^{3 / 4}}, \\
\xi_{21} & =\frac{1}{16}\left\{\left(g_{1}\right)_{\tilde{u} \tilde{u} \tilde{u}}+\left(g_{1}\right)_{\tilde{u} \tilde{v} \tilde{v}}+\left(g_{2}\right)_{\tilde{u} \tilde{u} \tilde{v}}+\left(g_{2}\right)_{\tilde{v} \tilde{v} \tilde{v}}+i\left[\left(g_{2}\right)_{\tilde{u} \tilde{u} \tilde{u}}+\left(g_{2}\right)_{\tilde{u} \tilde{v} \tilde{v}}-\left(g_{1}\right)_{\tilde{u} \tilde{u} \tilde{v}}-\left(g_{1}\right)_{\tilde{v} \tilde{v} \tilde{v}}\right]\right\} \\
& =\frac{\left(\sqrt{4 \bar{x}^{2}-f_{1}^{2}}+i f_{1}\right)\left(\bar{x}^{3}\left(f_{3} \bar{x}+3 f_{2}\right)+f_{1}\left(1-3 f_{2}\right) \bar{x}^{2}-3 f_{1}^{2} \bar{x}+2 f_{1}^{3}\right)}{32 \bar{x}^{4}-8 f_{1}^{2} \bar{x}^{2}} .
\end{aligned}
$$

The above normal form yields the approximation

$$
\zeta \rightarrow \lambda \zeta+c_{1} \zeta^{2} \bar{\zeta}+O\left(|\zeta|^{4}\right)
$$

with $c_{1}=i \lambda \alpha_{1}$ and $\alpha_{1}$ being the first twist coefficient.

The coefficient $c_{1}$ can be computed directly using the formula

$$
c_{1}=\frac{\xi_{20} \xi_{11}(\bar{\lambda}+2 \lambda-3)}{\left(\lambda^{2}-\lambda\right)(\bar{\lambda}-1)}+\frac{\left|\xi_{11}\right|^{2}}{1-\bar{\lambda}}+\frac{2\left|\xi_{02}\right|^{2}}{\lambda^{2}-\bar{\lambda}}+\xi_{21}
$$


derived by Wan in the context of Hopf bifurcation theory [34]. In [26] it is shown that when one uses area-preserving coordinate changes Wan's formula yields the twist coefficient $\alpha_{1}$ that is used to verify the non-degeneracy condition necessary to apply the KAM theorem.

By using

$$
\begin{aligned}
& \xi_{20} \xi_{11}=\frac{\left(\sqrt{4 \bar{x}^{2}-f_{1}^{2}}+i f_{1}\right)^{2}\left(f_{2} \bar{x}^{2}+f_{1} \bar{x}-f_{1}^{2}\right)^{2}}{16 \bar{x}^{3}\left(4 \bar{x}^{2}-f_{1}^{2}\right)^{3 / 2}}, \\
& \xi_{11} \overline{\xi_{11}}=\frac{\left(f_{2} \bar{x}^{2}+f_{1} \bar{x}-f_{1}^{2}\right)^{2}}{2 \bar{x}\left(4 \bar{x}^{2}-f_{1}^{2}\right)^{3 / 2}} \\
& \xi_{02} \overline{\xi_{02}}=\frac{\left(f_{2} \bar{x}^{2}+f_{1} \bar{x}-f_{1}^{2}\right)^{2}}{8 \bar{x}\left(4 \bar{x}^{2}-f_{1}^{2}\right)^{3 / 2}}
\end{aligned}
$$

a straightforward calculation yields

$$
\begin{aligned}
c_{1} & =\frac{\xi_{20} \xi_{11}(\bar{\lambda}+2 \lambda-3)}{\left(\lambda^{2}-\lambda\right)(\bar{\lambda}-1)}+\frac{\left|\xi_{11}\right|^{2}}{1-\bar{\lambda}}+\frac{2\left|\xi_{02}\right|^{2}}{\lambda^{2}-\bar{\lambda}}+\xi_{21} \\
& =\Theta(\bar{x}) \frac{\bar{x}^{4}\left(2 f_{3} \bar{x}+f_{2}\left(f_{2}+6\right)\right)+f_{1} \bar{x}^{3}\left(f_{3} \bar{x}+f_{2}\left(2 f_{2}-1\right)+2\right)-f_{1}^{2} \bar{x}^{2}\left(f_{3} \bar{x}+4 f_{2}+4\right)-f_{1}^{3} f_{2} \bar{x}+2 f_{1}^{4}}{4 \bar{x}\left(f_{1}-2 \bar{x}\right)^{2}\left(\bar{x}+f_{1}\right)\left(2 \bar{x}+f_{1}\right)\left(-i \sqrt{4 \bar{x}^{2}-f_{1}^{2}}+2 \bar{x}+f_{1}\right)},
\end{aligned}
$$

where

$$
\Theta(\bar{x}):=f_{1}\left(\sqrt{4 \bar{x}^{2}-f_{1}^{2}}+i \bar{x}\right)+\bar{x}\left(\sqrt{4 \bar{x}^{2}-f_{1}^{2}}-2 i \bar{x}\right)+i f_{1}^{2} .
$$

It can be proved that

$$
\begin{aligned}
\alpha_{1} & =-i \bar{\lambda} c_{1} \\
& =\frac{2 f_{3} \bar{x}^{5}+\left(f_{2}\left(f_{2}+6\right)+f_{1} f_{3}\right) \bar{x}^{4}-f_{1}\left(-2 f_{2}^{2}+f_{2}+f_{1} f_{3}-2\right) \bar{x}^{3}-4 f_{1}^{2}\left(f_{2}+1\right) \bar{x}^{2}-f_{1}^{3} f_{2} \bar{x}+2 f_{1}^{4}}{4\left(f_{1}-2 \bar{x}\right)^{2}\left(\bar{x}+f_{1}\right)\left(2 \bar{x}+f_{1}\right)},
\end{aligned}
$$

which implies that $\alpha_{1} \neq 0$ if (13) holds.

Since map (9) is exponentially equivalent to an area-preserving map $F$, an immediate consequence of Theorems 1 and 2 is the following result.

Theorem 3 Let $\bar{x}>0$ be the equilibrium point of (1) such that $\left|f^{\prime}(\bar{x})\right|<2 \bar{x}$. If (13) holds, then there exist periodic points of $T$ with arbitrarily large period in every neighborhood of $(\bar{x}, \bar{x})$. In addition, $\bar{x}$ is a stable equilibrium point of (1).

\section{Examples}

In this section, we apply Theorem 3 to several difference equations of the form (1) that have been listed in Sect. 1.

4.1 Example 1: $x_{n+1}=\frac{x_{n}^{k}+a}{x_{n}^{p} x_{n-1}}$

The equation

$$
x_{n+1}=\frac{x_{n}^{k}+a}{x_{n}^{p} x_{n-1}}
$$


where $k, p, a$ and the initial conditions $x_{0}, x_{1}$ are positive, is analyzed in [12] with fixed the value of $a$ as $a=\left(2^{k-p-2}-1\right) / 2^{k}$, where $k>p+2$ and $p \geq 1$.

Now, we assume that $a$ is any positive real number.

The equilibrium point of Equation (16) satisfies

$$
\bar{x}^{p+2}=\bar{x}^{k}+a .
$$

Similar as in Proposition 2.2 [12] one can prove the following.

Proposition 1 Assume that $k, p$, and a are positive. Let $x_{0}$ be the positive solution of the equation $x_{0}^{k-p-2}=(p+2) / k$, and let $y_{0}=x_{0}^{p+2}-x_{0}^{k}$. Then the following holds:

(a) If $k>p+2$, then for $0<a<y_{0}$ Equation (16) has exactly two positive equilibrium points, for $a=y_{0}$ it has exactly one, and for $a>y_{0}$ it has none.

(b) If $k<p+2$, then Equation (16) has exactly one positive equilibrium point.

Since

$$
f^{\prime}(\bar{x})-2 \bar{x}=-\frac{\left(p \bar{x}^{k}-k \bar{x}^{k}+2 \bar{x}^{p+2}+a p\right)}{\bar{x}^{p+1}}
$$

and

$$
f^{\prime}(\bar{x})+2 \bar{x}=-\frac{\left(p \bar{x}^{k}-k \bar{x}^{k}-2 \bar{x}^{p+2}+a p\right)}{\bar{x}^{p+1}},
$$

we have that if $\bar{x}>0$ then $\left|f^{\prime}(\bar{x})\right|<2 \bar{x}$ if and only if

$$
(k-p-2) \bar{x}^{k}<a(p+2) \text { and }(k-p+2) \bar{x}^{k}>a(p-2) .
$$

Hence, $\bar{x}$ is an elliptic point if and only if condition (17) is satisfied.

A tedious symbolic computation done with package Mathematica yields

$$
\alpha_{1}=\frac{a k^{3} \bar{x}^{k}\left((k-p-2)(k-p+1) \bar{x}^{2 k}+2 a k \bar{x}^{k}-a^{2}\left(p^{2}+p-2\right)\right)}{4\left((-k+p-2) \bar{x}^{k}+a(p-2)\right)\left((-k+p-1) \bar{x}^{k}+a(p-1)\right)\left((-k+p+2) \bar{x}^{k}+a(p+2)\right)^{2}} .
$$

Therefore we have the following statement.

Theorem 4 Assume that $k, p$, and $a$ are positive and the initial conditions $x_{0}, x_{1}$ are positive. Let $\bar{x}>0$ be an equilibrium point of (16) and $T$ be the map associated with Equation (16). Then $(\bar{x}, \bar{x})$ is an elliptic fixed point of $T$ if and only if

$$
(k-p-2) \bar{x}^{k}<a(p+2) \text { and }(k-p+2) \bar{x}^{k}>a(p-2) .
$$

Further, if

$$
(k-p-2)(k-p+1) \bar{x}^{2 k}+2 a k \bar{x}^{k}-a^{2}\left(p^{2}+p-2\right) \neq 0,
$$

then there exist periodic points of the map $T$ with arbitrarily large period in every neighborhood of $(\bar{x}, \bar{x})$. In addition, $\bar{x}$ is a stable equilibrium point of (16). 

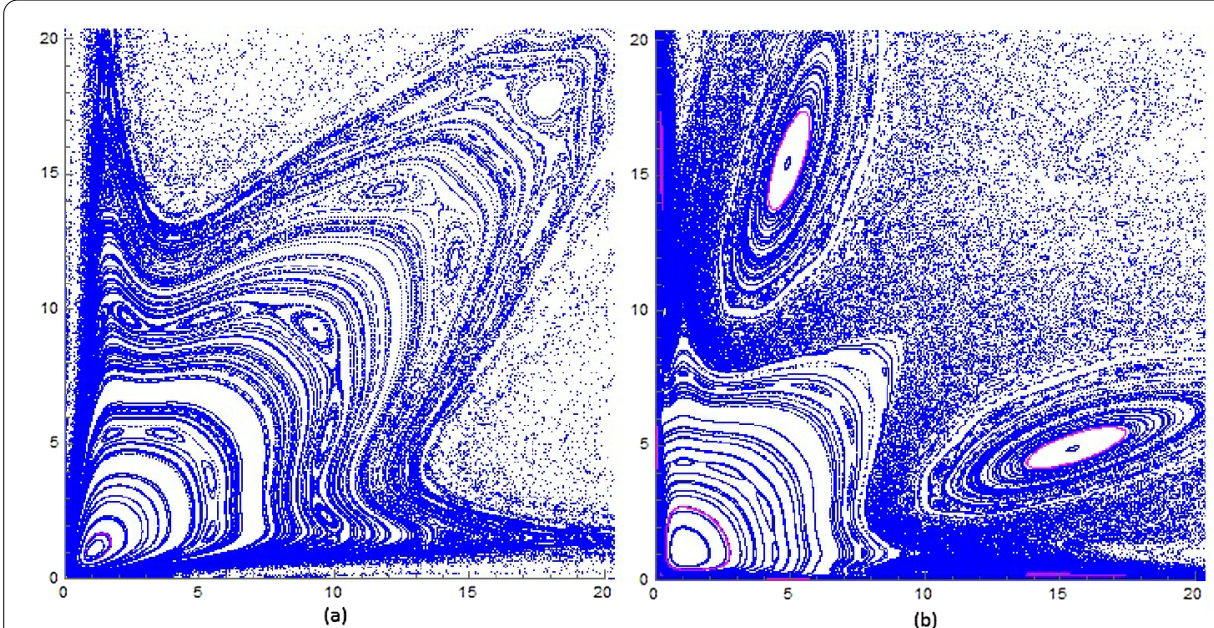

Figure 1 Some orbits of the map $T$ associated with Eq. (16) for (a) $k=2.1, p=1$, and $a=0.1$ and (b) $k=2.01$, $p=2$, and $a=0.1$

Figure 1 shows phase portraits of the orbits of the map $T$ associated with Equation (16) for some values of the parameters $p, k$, and $a$. Neither of these two plots shows any selfsimilarity character.

4.2 Example 2: $x_{n+1}=\frac{A+B x_{n}+C x_{n}^{2}}{\left(D+E x_{n}\right) x_{n-1}}$

The equation

$$
x_{n+1}=\frac{A+B x_{n}+C x_{n}^{2}}{\left(D+E x_{n}\right) x_{n-1}},
$$

where $A, B, C, D$, and $E$ are nonnegative and the initial conditions $x_{0}, x_{1}$ are positive, is analyzed by using the methods of algebraic and projective geometry in $[4,5]$ where $C=D$ and $E=1$ and by using KAM theory in [8] where $C=D=1$ and $A, B, E>0$. Now, we assume that

$$
(D, E>0 \wedge A+B>0) \vee(D, E>0 \wedge A+B=0 \wedge C>D) .
$$

The equilibrium point of Equation (18) satisfies

$$
E \bar{x}^{3}-\bar{x}^{2}(C-D)-B \bar{x}-A=0 .
$$

By using Descartes' rule of sign, we obtain that this equation has one positive root. Therefore, Equation (18) has one positive equilibrium point.

If $D, E>0$, then the change $x_{n}=\frac{D}{E} y_{n}$ conjugates Equation (18) to

$$
y_{n+1}=\frac{a+b y_{n}+c y_{n}^{2}}{\left(1+y_{n}\right) y_{n-1}},
$$

where the parameters $a, b$, and $c$ are

$$
a=\frac{A E^{2}}{D^{3}}, \quad b=\frac{B E}{D^{2}} \quad \text { and } \quad c=\frac{C}{D} .
$$


One can see that the following holds.

Proposition 2 Assume $a, b, c \geq 0$ and $a+b>0$ or $a+b=0 \wedge c>1$. Let $\bar{x}$ be a positive equilibrium of Equation (19), then $\left|f^{\prime}(\bar{x})\right|<2 \bar{x}$.

A tedious symbolic computation done with package Mathematica yields

$$
\alpha_{1}=\frac{\Gamma_{1}+\Gamma_{2} \bar{x}+\Gamma_{3} \bar{x}^{2}}{2(\bar{x}+1)^{2}(2 c \bar{x}+\bar{x}+b)\left(2 \bar{x}(b-c+1)+(c+2) \bar{x}^{2}+3 a-b\right)^{2}\left(2 \bar{x}(b+c+1)+(3 c+2) \bar{x}^{2}+a+b\right)},
$$

where

$$
\begin{aligned}
& \Gamma_{1}=a^{3} b^{2}+25 a^{3} b c^{2}+66 a^{3} b c+11 a^{3} b+20 a^{3} c^{3}+70 a^{3} c^{2}+55 a^{3} c-a^{3}-2 a^{2} b^{3} \\
& -12 a^{2} b^{2} c^{3}+5 a^{2} b^{2} c^{2}-8 a^{2} b^{2} c-5 a^{2} b^{2}-29 a^{2} b c^{5}-44 a^{2} b c^{4}-82 a^{2} b c^{3} \\
& -46 a^{2} b c^{2}+22 a^{2} b c+2 a^{2} b-8 a^{2} c^{7}-8 a^{2} c^{6}-16 a^{2} c^{5}-2 a^{2} c^{4}+8 a^{2} c^{2}+8 a^{2} c \\
& +3 a b^{4} c^{2}+8 a b^{4} c+a b^{4}+a b^{3} c^{4}+16 a b^{3} c^{3}-6 a b^{3} c^{2}-2 a b^{3} c-7 a b^{3}-3 a b^{2} c^{6} \\
& +10 a b^{2} c^{5}-26 a b^{2} c^{4}-3 a b^{2} c^{3}+a b^{2} c^{2}-5 a b^{2} c-a b^{2}-a b c^{8}+6 a b c^{7}-14 a b c^{6} \\
& +8 a b c^{5}+a b c^{4}+a c^{9}-3 a c^{8}+3 a c^{7}-a c^{6}+b^{4}, \\
& \Gamma_{2}=11 a^{3} b c+4 a^{3} b+8 a^{3} c^{3}+63 a^{3} c^{2}+54 a^{3} c+a^{3}+24 a^{2} b^{2} c^{2}+75 a^{2} b^{2} c+16 a^{2} b^{2} \\
& -20 a^{2} b c^{4}-18 a^{2} b c^{3}+18 a^{2} b c^{2}+110 a^{2} b c+6 a^{2} b-8 a^{2} c^{6}-17 a^{2} c^{5}-33 a^{2} c^{4} \\
& -35 a^{2} c^{3}+21 a^{2} c^{2}+37 a^{2} c-a^{2}+a b^{4} c-a b^{4}-10 a b^{3} c^{3}+18 a b^{3} c^{2}-a b^{3} c-19 a b^{3} \\
& -31 a b^{2} c^{5}-38 a b^{2} c^{4}-95 a b^{2} c^{3}-54 a b^{2} c^{2}-15 a b^{2} c-6 a b^{2}-9 a b c^{7}-4 a b c^{6} \\
& -25 a b c^{5}-3 a b c^{4}-4 a b c^{2}+8 a b c+a b+a c^{8}-2 a c^{7}+a c^{6}+3 b^{5} c^{2}+8 b^{5} c+b^{5} \\
& +b^{4} c^{4}+16 b^{4} c^{3}-6 b^{4} c^{2}-2 b^{4} c-b^{4}-3 b^{3} c^{6}+10 b^{3} c^{5}-26 b^{3} c^{4}-3 b^{3} c^{3}+b^{3} c^{2} \\
& +2 b^{3} c-b^{3}-b^{2} c^{8}+6 b^{2} c^{7}-14 b^{2} c^{6}+8 b^{2} c^{5}+b^{2} c^{4}+b c^{9}-3 b c^{8}+3 b c^{7}-b c^{6}, \\
& \Gamma_{3}=16 a^{3} c^{2}+19 a^{3} c+a^{3}+12 a^{2} b^{2} c+8 a^{2} b^{2}+22 a^{2} b c^{3}+92 a^{2} b c^{2}+84 a^{2} b c+6 a^{2} b \\
& -8 a^{2} c^{5}-6 a^{2} c^{4}-10 a^{2} c^{3}+33 a^{2} c^{2}+28 a^{2} c-a^{2}-a b^{4}+a b^{3} c^{2}+15 a b^{3} c-7 a b^{3} \\
& -33 a b^{2} c^{4}-16 a b^{2} c^{3}-65 a b^{2} c^{2}-25 a b^{2} c-6 a b^{2}-38 a b c^{6}-30 a b c^{5}-78 a b c^{4} \\
& -7 a b c^{3}+5 a b c^{2}+9 a b c+a b-8 a c^{8}+a c^{7}-9 a c^{6}+14 a c^{5}+2 a c^{4}+b^{5} c+b^{5} \\
& +5 b^{4} c^{3}+18 b^{4} c^{2}-b^{4}-b^{3} c^{5}+21 b^{3} c^{4}-35 b^{3} c^{3}-4 b^{3} c^{2}+b^{3} c-b^{3}-4 b^{2} c^{7} \\
& +17 b^{2} c^{6}-45 b^{2} c^{5}+22 b^{2} c^{4}+4 b^{2} c^{3}-b c^{9}+8 b c^{8}-22 b c^{7}+23 b c^{6}-7 b c^{5}-b c^{4} \\
& +c^{10}-4 c^{9}+6 c^{8}-4 c^{7}+c^{6} \text {. }
\end{aligned}
$$

In Table 1 we compute the twist coefficient for some values $a, b, c \geq 0$.

Theorem 5 Assume that $a, b$, and $c$ are positive numbers such that $a+b>0$. Let $\bar{x}>0$ be an equilibrium point of Equation (19) and $T$ be the map associated with Equation (19). Then $(\bar{x}, \bar{x})$ is an elliptic fixed point of $T$ and, if $\alpha_{1} \neq 0$, then there exist periodic points of the map $T$ with arbitrarily large period in every neighborhood of $(\bar{x}, \bar{x})$. In addition, $\bar{x}$ is a stable equilibrium point of (19). 
Table 1 The first twist coefficient for some values of $a, b, c \geq 0$

\begin{tabular}{lll}
\hline Parameters & Equation & The first twist coefficient \\
\hline$a=b=0, c>1, \bar{x}=c-1$ & $y_{n+1}=\frac{c y_{n}^{2}}{\left(1+y_{n}\right) y_{n-1}}$ & $\alpha_{1}=\frac{c}{12 c^{2}+10 c+2}(b-1) b$ \\
$a=c=0, \bar{x}=\frac{1}{2}(\sqrt{4 b+1}-1)$ & $y_{n+1}=\frac{b}{\left(1+y_{n}\right) y_{n-1}}$ & $\alpha_{1}=\frac{a}{2(4 b+1)(3(\sqrt{4 b+1}+1)+b(\sqrt{4 b+1}+6))}$ \\
$b=c=0, a=\bar{x}^{3}+\bar{x}^{2}$ & $y_{n+1}=\frac{a}{\left(1+y_{n}\right) y_{n-1}}$ & $\alpha_{1}=\frac{(\bar{x}-1)(\bar{x} \bar{x}+2)^{2}}{2(\bar{x}+2)}$ \\
$c=0, a=\bar{x}^{3}+\bar{x}^{2}-b \bar{x}$ & $y_{n+1}=\frac{a+b y_{n}}{\left(1+y_{n}\right) y_{n-1}}$ & $\alpha_{1}=\frac{\left(b-\bar{x}^{2}\right)\left(2 b^{3}-4 b \bar{x}^{4}-(b(b+5)-1) \bar{x}^{3}+(b-2) b \bar{x}^{2}-\bar{x}^{5}+b^{3}\right)}{2(\bar{x}+1)(\bar{x}+b)(\bar{x}(\bar{x}+2)+b)(b-\bar{x}(3 \bar{x}+2))^{2}}$ \\
\hline
\end{tabular}

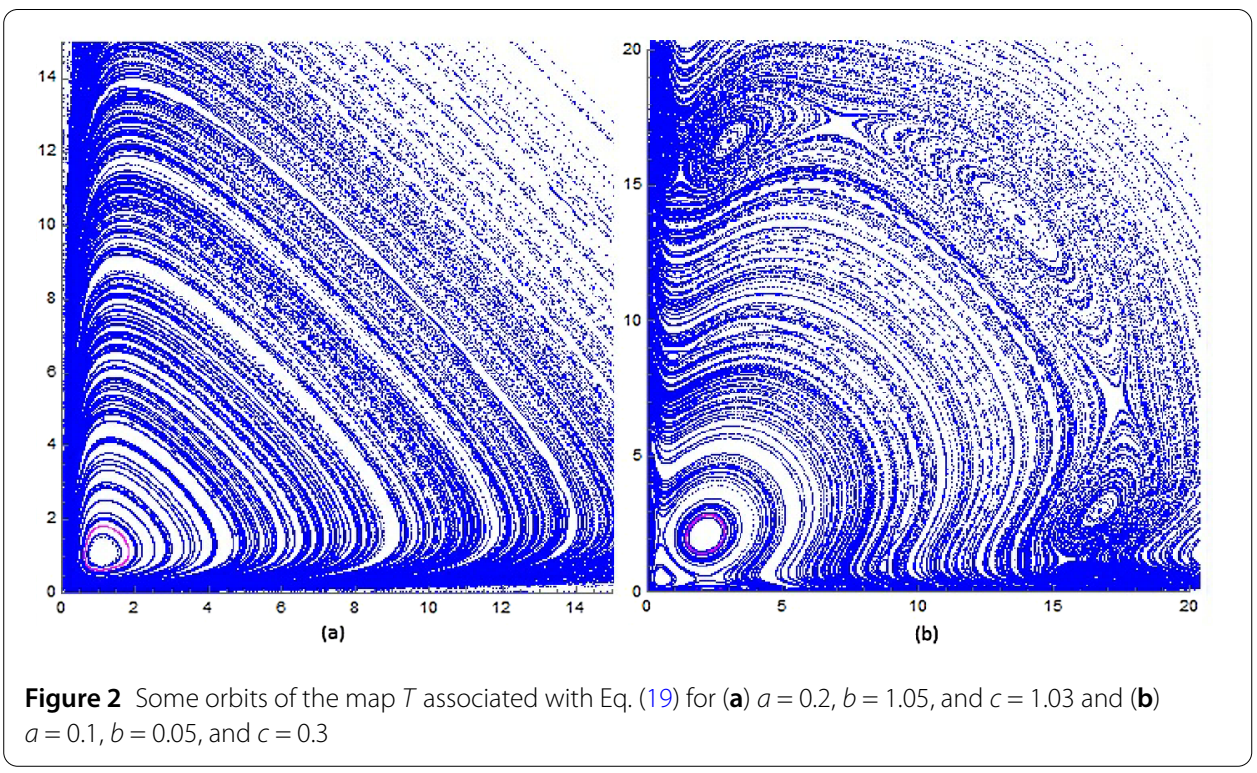

Figure 2 shows phase portraits of the orbits of the map $T$ associated with Equation (19) for some values of the parameters $a, b$, and $c$.

\subsection{Example 3: $x_{n+1}=\frac{a+b x_{n}+c x_{n}^{2}}{x_{n-1}}$}

In $[4,5]$ the authors analyzed the equation

$$
x_{n+1}=\frac{a+b x_{n}+c x_{n}^{2}}{x_{n-1}}
$$

where $a, b$, and $c$ are nonnegative and the initial conditions $x_{0}, x_{1}$ are positive, by using the methods of algebraic and projective geometry where $c=1$. It is easy to see that Equation (20) has one positive equilibrium

$$
\bar{x}=\frac{b+\sqrt{4 a c+4 a+b^{2}}}{2(1-c)}
$$

for $c<1$. Further, $\left|f^{\prime}(\bar{x})\right|-2 \bar{x}=-\sqrt{b^{2}+4 a(1-c)}<0$.

By using package Mathematica, we obtain

$$
\alpha_{1}=\frac{16 a^{2}(c-1)^{2} c(c+1)+a b^{2}\left(-8 c^{3}+8 c^{2}+c-1\right)+b \Gamma_{4} \sqrt{-4 a c+4 a+b^{2}}+b^{4}\left(c^{2}-c+1\right)}{2\left(b^{2}-4 a c+4 a+\right)\left(2 b+(c+1) \sqrt{b^{2}-4 a c+4 a}\right)\left(3 b+(2 c+1) \sqrt{b^{2}-4 a c+4 a}\right)},
$$




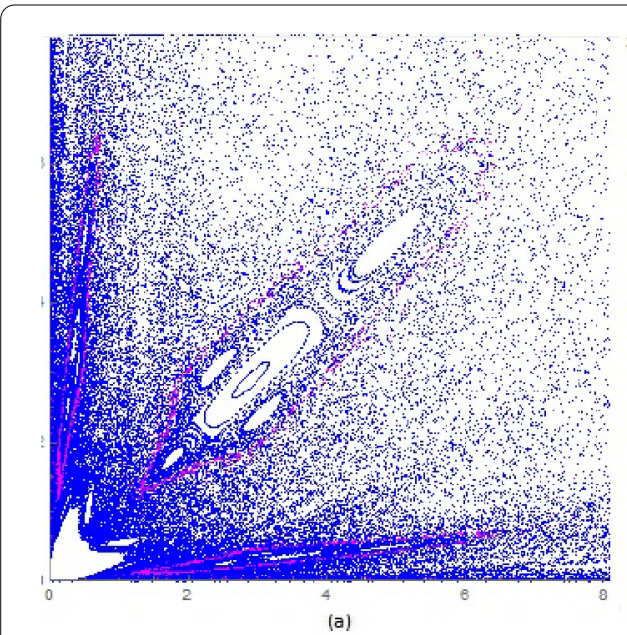

(a)

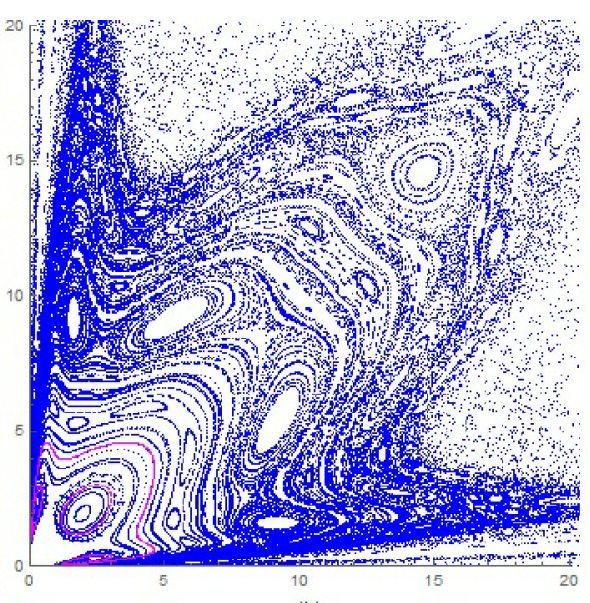

(b)

Figure 3 Some orbits of the map $T$ associated with Eq. (20) for (a) $a=0.1, b=0.002$, and $c=0.001$ and (b) $a=0.1, b=0.02$, and $c=0.001$

where

$$
\Gamma_{4}=a\left(4 c^{3}-12 c^{2}+7 c+1\right)-b^{2}\left(c^{2}-3 c+1\right) .
$$

Theorem 6 Assume that $a, b$, and c are positive numbers such that $a+b>0$ and $c<1$. Let $\bar{x}>0$ be the equilibrium point of Equation (20) and $T$ be the map associated with Equation (20). Then $(\bar{x}, \bar{x})$ is an elliptic fixed point of $T$ and, if $\alpha_{1} \neq 0$, there exist periodic points with arbitrarily large period in every neighborhood of $(\bar{x}, \bar{x})$. In addition, $\bar{x}$ is a stable equilibrium point of (20).

Figure 3 shows phase portraits of the orbits of the map $T$ associated with Equation (20) for some values of the parameters $a, b$, and $c$.

\section{Conclusion}

In this paper, we investigated the stability of a class of difference equations of the form $x_{n+1}=\frac{f\left(x_{n}\right)}{x_{n-1}}, n=0,1, \ldots$ We assume that the function $f$ is sufficiently smooth and the initial conditions are arbitrary positive real numbers. It is enough to assume that the function $f$ is in $C^{(3)}(0,+\infty)$. We show how the map $T$ associated with this difference equation leads to diffeomorphism $F$. We prove some properties of the map $F$, and we establish the condition under which an equilibrium point $(0,0)$ in $u, v$ coordinates is an elliptic fixed point. Also, we compute the first twist coefficient. The condition for an elliptic fixed point to be nondegenerate and non-resonant is established in closed form. This condition depends only on the values of the first, second, and third derivatives of the function $f$ at the equilibrium point. We apply our result to several difference equations that have been investigated by others. By numerical computations, we confirm our analytic results.

\section{Acknowledgements}

The authors are thankful to the anonymous referees for their helpful comments and the editor for constructive suggestions to improve the paper in current form. 
Availability of data and materials

Not applicable.

Ethics approval and consent to participate

Not applicable.

Competing interests

The authors declare that they have no competing interests.

Authors' contributions

All authors contributed equally and significantly in writing this article. All authors read and approved the final manuscript.

\section{Author details}

'Department of Mathematics, Faculty of Science, University of Sarajevo, Sarajevo, Bosnia and Herzegovina. ${ }^{2}$ Faculty of Electrical Engineering, University of Sarajevo, Sarajevo, Bosnia and Herzegovina.

\section{Publisher's Note}

Springer Nature remains neutral with regard to jurisdictional claims in published maps and institutional affiliations.

Received: 22 March 2019 Accepted: 20 May 2019 Published online: 29 May 2019

\section{References}

1. Amleh, A.M., Camouzis, E., Ladas, G.: On the dynamics of a rational difference equation, part 1. Int. J. Difference Equ. 3(1), 1-35 (2008)

2. Barbeau, E., Gelbord, B., Tanny, S.: Periodicity of solutions of the generalized Lyness recursion. J. Differ. Equ. Appl. 1 291-306 (1995)

3. Bastien, G., Rogalski, M.: Global behavior of the solutions of Lyness' difference equation $u_{n+2} u_{n}=u_{n+1}+a$. J. Differ. Equ. Appl. 10, 977-1003 (2004)

4. Bastien, G., Rogalski, M.: On some algebraic difference equations $u_{n+2} u_{n}=g\left(u_{n+1}\right)$ related to families of conics or cubics: generalization of the Lyness' sequences. J. Math. Anal. Appl. 300, 303-333 (2004)

5. Bastien, G., Rogalski, M.: On the algebraic difference equations $u_{n+2} u_{n}=\psi\left(u_{n+1}\right)$ in $\mathbb{R}_{*}^{+}$, related to a family of elliptic quartics in the plane. Adv. Differ. Equ. 2005, 948567 (2005)

6. Beukers, F., Cushman, R.: Zeeman's monotonicity conjecture. J. Differ. Equ. 143, 191-200 (1998)

7. Denette, E., Kulenović, M.R.S., Pilav, E.: Birkhoff normal forms, KAM theory and time reversal symmetry for certain rational map. Mathematics 4(1), 20 (2016)

8. Garic-Demirovic, M., Nurkanovic, M., Nurkanovic, Z.: Stability, periodicity, and symmetries of certain second-order fractional difference equation with quadratic terms via KAM theory. Math. Methods Appl. Sci. 40, 306-318 (2017)

9. Gidea, M., Meiss, J.D., Ugarcovici, I., Weiss, H.: Applications of KAM theory to population dynamics. J. Biol. Dyn. 5(1), 44-63 (2011)

10. Grove, E.A., Janowski, E.J., Kent, C.M., Ladas, G.: On the rational recursive sequence $x_{n+1}=\frac{\alpha x_{n}+\beta}{\left(\gamma x_{n}+\delta\right) x_{n-1}}$. Commun. Appl. Nonlinear Anal. 1(13), 61-72 (1994)

11. Hale, J.K., Kocak, H.: Dynamics and Bifurcation. Springer, New York (1991)

12. Haymond, R.E., Thomas, E.S.: Phase portraits for a class of difference equations. J. Differ. Equ. Appl. 5, 177-202 (1999)

13. Jašarević-Hrustić, S., Kulenović, M.R.S., Nurkanović, Z., Pilav, E.: Birkhoff normal forms, KAM theory and symmetries for certain second order rational difference equation with quadratic terms. J. Differ. Equ. 10(2), 181-199 (2015)

14. Kocic, V.L., Ladas, G.: Global Behavior of Nonlinear Difference Equations of Higher Order with Applications. Kluwer Academic, Dordreht (1993)

15. Kocic, V.L., Ladas, G., Rodrigues, I.W.: On the rational recursive sequences. J. Math. Anal. Appl. 173, 127-157 (1993)

16. Kocic, V.L., Ladas, G., Tzanetopoulos, G., Thomas, E.: On the stability of Lyness equation. In: Dynamics of Continuous, Discrete and Impulsive Systems (1), pp. 245-254 (1995)

17. Kulenović, M.R.S.: Invariants and related Liapunov functions for difference equations. Appl. Math. Lett. 13, 1-8 (2000)

18. Kulenović, M.R.S., Ladas, G.: Dynamics of Second Order Rational Difference Equations: With Open Problems and Conjectures. Chapman and Hall/CRC, London (2001)

19. Kulenović, M.R.S., Merino, O.: Discrete Dynamical Systems and Difference Equations with Mathematica. Chapman Hall/CRC, Boca Raton (2002)

20. Kulenović, M.R.S., Nurkanović, Z.: Stability of Lyness equation with period three coefficient. Rad. Mat. 12, 153-161 (2004)

21. Kulenović, M.R.S., Nurkanović, Z.: Stability of Lyness equation with period-two coefficient via KAM theory. J. Concr. Appl. Math. 6, 229-245 (2008)

22. Ladas, G., Tzanetopoulos, G., Tovbis, A.: On May's host parasitoid model. J. Differ. Equ. Appl. 2, 195-204 (1996)

23. May, R.M.: Host-parasitoid system in patchy environments. A phenomenological model. J. Anim. Ecol. 47, 833-843 (1978)

24. May, R.M., Hassel, M.P.: The dynamics of multiparasitoid host interactions. Am. Nat. 117, 234-261 (1981)

25. Mestel, B.D.: On globally periodic solutions of the difference equation $x_{n+1}=\frac{f\left(x_{n}\right)}{x_{n-1}}$. J. Differ. Equ. Appl. 3, 201-209 (2001)

26. Moeckel, R.: Generic bifurcations of the twist coefficient. Ergod. Theory Dyn. Syst. 10(1), 185-195 (1990)

27. Moser, J.: On invariant curves of area-preserving mappings of an annulus. Nachr. Akad. Wiss. Gött., 2 1962, 1-20 (1962)

28. Nurkanović, M., Nurkanović, Z.: Birkhoff normal forms, KAM theory, periodicity and symmetries for certain rational difference equation with cubic terms. Sarajevo J. Math. 25, 217-231 (2016)

29. Siegel, C.L., Moser, J.K.: Lectures on Celestial Mechanics. Springer, New York (1971) 
30. Siezer, W.: Periodicity in the May's host parasitoid equation. In: Advances Studies in Pure Mathematics 53 (2009)

31. Sternberg, S.: Celestial Mechanics. II. W. A. Benjamin, New York (1969)

32. Tabor, M.: Chaos and Integrability in Nonlinear Dynamics. An Introduction. Wiley, New York (1989)

33. Taylor, A.D.: Aggregation, competition, and host-parasitoid dynamics: stability conditions don't tell it all. Am. Nat. 141, 501-506 (1993)

34. Wan, Y.H.: Computation of the stability condition for the Hopf bifurcation of diffeomorphisms on $\mathcal{R}^{2}$. SIAM J. Appl. Math. 34(1), 167-175 (1978)

35. Zeeman, E.C.: Geometric Unfolding of a Difference Equation. Hertford College, Oxford (1996)

Submit your manuscript to a SpringerOpen ${ }^{\mathcal{O}}$ journal and benefit from:

- Convenient online submission

- Rigorous peer review

- Open access: articles freely available online

- High visibility within the field

- Retaining the copyright to your article

Submit your next manuscript at $\gg$ springeropen.com 